\title{
Organización metodológica de una unidad didáctica de educación física en tiempos del covid-19 \\ Methodological organization of a physical education didactic unit during covid-19
}

\author{
*Vicente Ramírez Arrabal, **Javier Herrero Robles, **Salvador Jiménez Marfil \\ *Universidad de Granada (España), **CEIP Valdelecrín (España)
}

\begin{abstract}
Resumen: Nos encontramos en los últimos meses en una situación excepcional, que ha cambiado seguramente para siempre muchos de los hábitos y de las formas de proceder que tenemos en nuestra vida diaria, y en nuestra vida profesional. Los profesionales de la educación en general y de la educación física en particular, se han tenido que adaptar y terminar el curso 2019/20 trabajando a distancia, usando formas creativas de enseñanza y vinculando el proceso de enseñanza-aprendizaje más que nunca a las tics, intentando que ese proceso no perdiera calidad. Para el curso 2020/21 el reto es el mismo, aunque en principio de manera presencial, presentándose una serie de condicionantes que tendremos que tener en cuenta para llevar a cabo una práctica segura. Este artículo aprovecha el programa estival de la Consejería de Educación y Deporte de la Junta de Andalucía (celebrado en julio de 2020) para anticiparse a toda esa serie de condicionantes, planteando en la introducción la problemática actual del Covid-19, que se une a los problemas habituales de sedentarismo que afectan a la población infantil y juvenil; enmarcado en un centro educativo con unas características determinadas y con un alumnado de sexto curso de primaria. La experiencia didáctica se basó en todas las recomendaciones que al respecto se habían dado, para llegar a unas propuestas (resultados-sesiones) concretas, con las que pudimos concluir con algunos cambios metodológicos a realizar en nuestro quehacer diario, para dar respuesta al Covid-19.
\end{abstract}

Palabras clave: Metodología, programa estival, Covid-19, unidad didáctica, educación física.

\begin{abstract}
We have found ourselves in an exceptional situation in recent months, which has surely forever changed many of the habits and ways of proceeding that we have in our daily life and in our professional life. Professionals in education in general and in physical education in particular, have had to adapt and finish the 2019/20 course working remotely, using creative ways of teaching and linking the teaching-learning process more than ever to tics, trying that this process did not lose quality. For the 2020/21 academic year the challenge is the same, although in principle in person, presenting a series of conditions that we will have to take into account to carry out a safe practice. This article takes advantage of the summer program of the Council of Education and Sports of the Junta de Andalucía (held in July 2020) to anticipate all this series of conditions, raising in the introduction the current problem of Covid-19, which joins the habitual problems of sedentary lifestyle that affect the child and youth population; framed in an educational center with certain characteristics and with sixth-grade primary school students. The didactic experience was based on all the recommendations that had been given in this regard, to arrive at specific proposals (results-sessions), with which we were able to conclude with some methodological changes to make in our daily work, to respond to the Covid- 19.
\end{abstract}

Keywords: Methodology, summer program, Covid-19, didactic unit, physical education.

\section{Introducción}

Las circunstancias en las que nos hemos visto envueltos los profesionales de la enseñanza en los últimos meses, han supuesto un cambio en la forma de concebir la Educación Física (EF) (Hortigüela-Alcalá, Garijo, y Pérez-Pueyo, 2021), y ha supuesto una prueba muy importante para el análisis y reflexión de la acción docente por parte de los maestros y profesores, ya que he-

Fecha recepción: 01-10-20. Fecha de aceptación: 24-05-21

Vicente Ramírez Arrabal

donvicenra@hotmail.com mos tenido que adaptar contenidos y competencias de una asignatura eminentemente práctica (Sierra-Díaz, González-Víllora, Toledo-Guijarro y Bermejo-Collada, 2021). La importancia que ha supuesto la brecha digital en muchos hogares de nuestra comunidad ha acrecentado en algunas situaciones las diferencias sociales entre alumnos y alumnas de nuestro entorno, ya que han sido muy importantes todas las metodologías relacionadas con las tics (Rivas, Gastélum-Cuadras, Velducea, Bustos y Esparza, 2021). La respuesta dada en cada caso, y más concretamente desde el área de EF, ha ayudado a que esta brecha sea menor en muchos casos. Una vez terminado el curso 2019/20, se planteó una nueva norma- 
lidad a los docentes para la vuelta al curso en septiembre, en la que nos encontramos con una serie de condicionantes nunca vistos anteriormente, a los que tuvimos que dar una respuesta adecuada. Desde este punto de vista y como docente del área de EF en la etapa de primaria, aprovechamos el programa de refuerzo estival de la Junta de Andalucía para poner en marcha una unidad didáctica enfocada a lo que con total seguridad encontraríamos en septiembre, intentando poner solución a los problemas con una de las armas más poderosas de un docente: los recursos metodológicos.

También hemos de tener en cuenta tal y como señala Márquez (2020), que la EF escolar es un equilibrador de la tendencia actual a la inactividad física; proporcionando además una adherencia y estilos de vida saludables (Zueck, García, Villalobos y Gutiérrez, 2020), que cada año aumenta con la edad tanto en niños como en adolescentes (Sánchez, del Pozo-Cruz, Rosa, Gómez y Barbosa, 2021), y que en el periodo de confinamiento no ha hecho más que incrementarse, teniendo en nuestro caso como docentes la necesidad de realizar una planificación adecuada a nivel competencial (Lleixá., Capllonch y González-Arévalo, 2015) en la vuelta a las aulas. Este autor alude a que el distanciamiento o aislamiento social, que tan importante es en la lucha contra la epidemia, ha contribuido por otra parte a favorecer la conducta sedentaria, por lo que tal y como se señala en medios como Clinical Trials (National Library of Medicine, 2020) ya existen múltiples registros que determinan el impacto real del confinamiento en diferentes grupos poblacionales, y no son precisamente positivos; entre ellos destacamos la disminución de capacidad en el aparato locomotor con pérdida de capacidad aeróbica, alteraciones metabólicas y de fuerza muscular, que en muchos casos requieren de varios meses de entrenamiento para recuperarla (McGuire et al. 2001). En esa misma línea Celis, Salas, Yáñez y Castillo (2020) evidencian que los períodos cortos de inactividad física inducida y el aumento de la conducta sedente tienen importantes implicaciones metabólicas en la salud, siendo nuestro país uno con las tasas más altas de sobrepeso y obesidad infantil a nivel europeo (Aranceta-Bartrina, Gianzo-Citores, y Pérez-Rodrigo, 2020). En este sentido encontramos ya evidencias de los efectos de la pandemia no solo a nivel fisiológico o metabólico sino también psicológico; tal y como señala Cabrera (2020) en población en edad infantil puede existir estrés postraumático, agresividad, rebeldía o desórdenes del sueño, aparte de obesidad como respuesta a la ansiedad derivada del confinamiento, también la restricción de movimiento provoca enfados, llantos, miedos, trastornos alimentarios y cierta hiperactividad. La cantidad de actividad física realizada es un factor determinante para compensar todas las alteraciones producidas y un factor que ha sido determinante para la fidelización a la práctica de actividad física durante el confinamiento (AlarcónMeza y Hall-López, 2020).

La propuesta realizada en este artículo se basa en varios documentos, algunos autonómicos, que versan sobre varios aspectos del área de EF en relación con la problemática del Covid-19 en la vuelta a las aulas, y que van en la línea del trabajo realizado por Baena-Morales, López-Morales y García-Taibo (2020). Destacamos dentro de estos uno muy interesante y que deberíamos tener en cuenta, que es el elaborado por el Colegio Oficial de Licenciados de Educación Física (COLEF), en el que se dan y se ofrecen medidas concretas de control y contingencia para la práctica de una EF segura y responsable ante la nueva normalidad. Obviamente también tenemos que destacar las orientaciones más o menos acertadas que al respecto ha dado la Consejería de Educación y Deporte de la Junta de Andalucía, a través del documento entregado a los docentes de EF en este periodo estival. En cualquier caso se han realizado adaptaciones, desdeñado y añadido medidas organizativas que creíamos necesarias e interesantes, en función del entorno en el que se ha llevado a cabo esta unidad didáctica; las cuales pueden ser muy interesantes para que el profesorado lo tenga en cuenta en la vuelta a las aulas. Entendemos también que obviamente son medidas dentro de un contexto concreto, y que cada docente tendrá que adoptar unas u otras en función principalmente de las características de su centro escolar, del material que disponga, del número de alumnado con los que trabaje, y en definitiva de los recursos con los cuales cuente.

Este artículo pretende proponer algunas soluciones a la problemática actual, teniendo como fin principal uno que ya contemplamos en el área de EF, como es la seguridad y la salud cuando practicamos actividad física.

\section{Contexto}

El contexto en el que se sitúa esta experiencia es algo singular, ya que el programa de refuerzo estival no se centra en el alumnado de un solo centro educativo, sino que al programa se puede adherir cualquier alumno o alumna del área de influencia del centro educativo. En concreto nos situamos en el CEIP Mario Vargas Llosa de la localidad de Marbella, en el que se han organizado 5 grupos de 8 a 10 niños y niñas cada uno, con edades 
comprendidas entre los 6 a los 12 años. El alumnado presenta características psico-evolutivas y socio-culturales heterogéneas, centrándonos en este artículo en las medidas metodológicas que se han tomado en el grupo concreto de alumnado de sexto curso (10 alumnos/as), aunque han sido muy similares en el resto de clases. El horario ha sido el habitual de 9 a 14 horas, distribuido en clases de inglés, matemáticas, lengua y educación física; en la cual hemos tenido en cuenta todos los factores que caracterizan este programa educativo en verano: meteorología, medidas covid-19, número de alumnado etc. El horario concreto para este grupo de sexto curso ha sido lunes y martes de 12 a 13 horas, miércoles de 10:30 a 11:30 horas, jueves de 9:30 a 10:30 horas y viernes de 13 a 14 horas. El horario ha sido un condicionante muy importante por las temperaturas, en las horas que eran después del recreo (a partir de las 11:30 horas). Se dispone de una amplia pista polideportiva que consta de dos canchas de baloncesto dentro de una pista de futbol-sala y una zona de patio techada en la que desarrollar la mayoría de los contenidos. En cualquier caso el espacio es amplio y suficiente para las necesidades de distancia y seguridad exigidas. La zona techada tiene pintados en el suelo diferentes juegos que serán muy útiles en diferentes partes de las sesiones y que se aprovecharán dada la escasez de otros materiales.

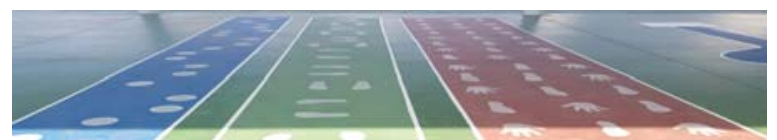

Figura1. Juegos pintados como recurso en varias partes de la sesión

El objetivo principal del programa de refuerzo es «participar en actividades de carácter deportivo que fomenten la motivación del alumnado, su participación y su adhesión a la realización de actividades en el entorno escolar,». Con esta intención se plantea esta propuesta físico-deportiva-educativa.

\section{Planteamiento didáctico}

Para la realización y puesta en práctica de esta unidad didáctica se cuenta con la normativa vigente, tanto el Decreto 97/2015 como la Orden 17 de Marzo de 2015, para el enfoque de los diferentes elementos curriculares, su programación y organización. En este sentido el primer documento con instrucciones concretas referidas a la nueva normalidad que se tuvo en cuenta, fue el que proporcionó la Consejería de Educación y Deporte de la Junta de Andalucía con el título: «Mate- rial didáctico de EF para primaria: programa de refuerzo estival». Dentro de este documento se concretan las orientaciones que más afectaban en la tarea diaria, adaptadas al contexto, a nuestra propia práctica educativa y al conocimiento que como docentes con más de 20 años de servicio se decidió aportar, modificar o eliminar. Entre estas destacamos:

-Mantener una serie de rutinas antes, durante y después de las actividades deportivas, así como acciones que colaboren al afianzamiento de esas rutinas en niños y niñas.

-Actividades variadas que abarcarán muchos contenidos propios del área de EF, excluyendo aquellos que puedan suponer contacto directo del alumnado entre ellos, o que los pongan en riesgo de contagio por la obligatoria y reglamentaria manipulación del material en determinados deportes, en cualquier caso, respetándose siempre los descansos y la hidratación.

-Planificación del trabajo diario, a fin de tener previsto posibles modificaciones sobre la unidad didáctica que se propone.

-Seguir las instrucciones relacionadas con el Covid19: aseo de manos y cara, cuidar el contacto con objetos y suelo, evitar tumbarse, respetar la distancia de seguridad...

-Alumnado que deberá llevar: mascarilla, guantes, una gorra, una botellita de agua, protector solar, comida saludable para el recreo, zapatillas adecuadas y ropa deportiva, incluso una segunda camiseta de recambio por si fuera necesario.

-Disponer de un termómetro por si se considera tomar la temperatura corporal de los niños/as.

-Provisión de un botiquín básico de primeros auxilios que contenga, además, algún sobrecito de azúcar.

Para la programación de la unidad didáctica también se tuvo en cuenta otro documento muy interesante, que en este caso fue elaborado por el COLEF, publicado en Junio de 2020, con el objetivo de minimizar los riesgos de contagio por Covid-19 en las clases de EF, denominado «Recomendaciones docentes para una educación física escolar segura y responsable ante la nueva normalidad». Este documento se resume y clasifica en dos apartados; por un lado las medidas curriculares, entre las que destaca la adaptación de las reglas de juegos y deportes para mantener las distancias de seguridad, con materiales que reducen el contacto y eliminando los implementos compartidos. Por otro las medidas de acceso; como la ubicación de los aseos, cercanos al alumnado, la disponibilidad del material higiénico, y dispensadores de gel desinfectante en diferentes puntos de la instalación. 
Otro estamento que ha elaborado un protocolo de actuación en sus diferentes equipos, para realizar una práctica deportiva que evite los contagios lo más segura posible, es la Federación Andaluza de Baloncesto (FAB). En el documento «Protocolo protección y prevención de la salud frente al Covid-19» con fecha de 21 de Julio de 2020, la dirección general de promoción del deporte, hábitos saludables y tejido deportivo de la Consejería de Educación y Deporte de la Junta de Andalucía autoriza a la FAB a la publicación de este documento, en el cual vemos destacable subrayar lo siguiente: se recogen una serie de medidas como el estornudar cubriéndose con un pañuelo, no compartir objetos, llevar las uñas cortas y cuidadas, evitando usar anillos, pulseras, relojes; evitar el uso de lentillas o recogerse el pelo largo son otras de las recomendaciones. Se potenciará el uso de carteles y señalización que fomente las medidas de higiene y prevención, distribuida por las zonas de práctica deportiva, entrada, aseos, vestuarios y principales zonas de paso.

En el mismo sentido destacar también las aportaciones realizadas por la Consejería de Educación de la Región de Murcia en el documento «medidas higiénicosanitarias en $E F$ ». Las instrucciones giran en torno a aspectos metodológicos concretos que envuelven la sesión de EF, y que nos pueden dar más pistas a la hora de concretar diferentes aspectos de la clase de EF. Aspectos como la necesidad de fomentar la composición de grupos estables a la hora de agrupar a los alumnos (parejas, cuartetos, sextetos...), o la disposición geométrica en filas-hileras, círculos, semicírculos o en damero; con la distancia requerida. Finalmente destacar que se propone utilizar la vía de comunicación visual y auditiva, haciendo hincapié en el feedback descriptivo y prescriptivo, que evite invasiones de espacios de los demás.

El último documento destacable que afronta esta problemática es el ofrecido por Pérez (2020), en el que desde su experiencia como técnico en riesgos laborales y como docente de Educación Secundaria realiza una serie de propuestas, como la necesidad de una planificación adecuada de todas las sesiones para una mayor satisfacción y motivación del alumnado, o evitar las actividades en espacios cerrados, delimitando zonas de entrada y de salida (Zueck, et al. 2020)

Con todas estas aportaciones, las propias del docente, y teniendo en cuenta el contexto, se concretaron una serie de medidas para dar respuesta a la situación actual. En cuanto a los aspectos metodológicos organizativos recogidos en esta experiencia, podemos señalar los siguientes, que se verán reflejados de una manera concreta en cada una de las sesiones:

-Juegos adaptados en sus normas y en su lógica interna para eliminar el contacto directo entre alumnado, intentando asegurar la distancia de seguridad.

-Generación de automatismos en la formación de las filas, teniendo en cuenta las nuevas señalizaciones. Tanto en las subidas como en las bajadas al patio el alumnado se sitúa siempre en el mismo lugar de la fila, a la misma distancia entre cada uno de ellos, y siguiendo siempre el mismo ritual para asegurarnos que mantienen la distancia de seguridad, llevando la mascarilla en todo momento en los traslados. Una vez en el patio cada uno tiene un espacio asignado donde colocar sus efectos personales (bolsa de aseo y botella de agua).

-Los objetivos que nos planteamos para esta unidad didáctica a partir de los criterios de evaluación son globales, intentando abarcar una gama de contenidos mayor que en el caso de unidades didácticas habituales, debido a la imposibilidad de llevar a cabo muchos de ellos por las circunstancias en las que nos encontramos. También estos elementos curriculares se deben enmarcar en el contexto estacional del verano y del programa estival en el que estamos trabajando.

-Rutinas en el calentamiento: aunque algunos de los juegos o ejercicios elegidos para realizar la parte inicial de las sesiones no se adecúe al resto de la sesión, optamos por realizar y enseñar 3 tipos de calentamientos diferentes en los que siempre haremos los mismos juegos o ejercicios, para que el alumnado los automatice, ganemos tiempo y nos aseguremos que se mantienen las normas de seguridad establecidas. Usamos espacios ya determinados en el centro como las huellas de calentamiento y calentamientos de tipo analítico y formal.

-En cuanto al material de clase intentamos por un lado tener el material suficiente para dos sesiones, siempre teniendo en cuenta que vamos a usar un material muy limitado en el caso de este programa de refuerzo estival, que se reduce a 20 aros, 10 pelotas de foam, 10 balones de baloncesto y a 8 conos. Este material es higienizado por el personal de limpieza del centro escolar en la hora del recreo a mitad de la mañana; y entre sesiones por el maestro. El material se coloca siempre en el mismo lugar, diferenciando el que se ha usado del que no, ya que el profesor entre sesiones lo higienizará dependiendo del uso que se le haya dado.

-Una vez que el alumnado llega al patio se sitúan en los espacios establecidos para cada uno, en las escaleras de acceso al edificio principal. En estas escaleras situaremos con tiza los espacios en los que el alumnado se 
puede sentar, y en los que no, asignando el mismo sitio para todo el mes a cada uno de los alumnos/as, y realizando la mayoría de las explicaciones en ese espacio. Una vez que se acaba un juego volvemos siempre al espacio asignado, bebemos agua y descansamos mientras atienden a la explicación del siguiente juego o ejercicio.

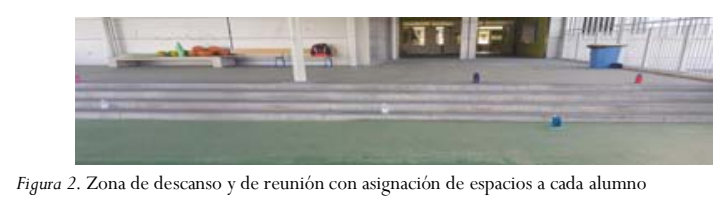

-Obviamente circunscribimos nuestra labor en torno a un material mínimo, teniendo un control exhaustivo del mismo en todo momento.
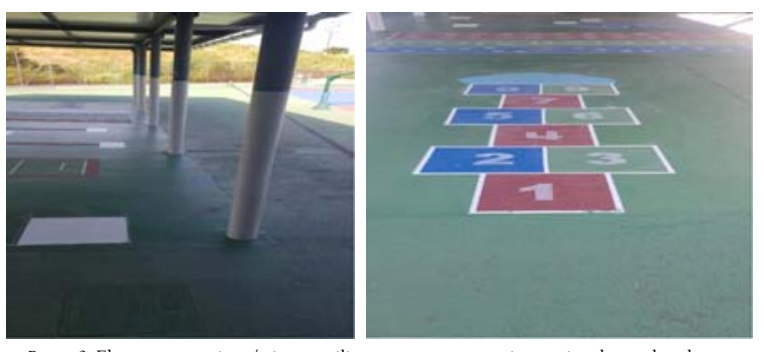

Figura 3. Elementos arquitectónicos a utilizar como recurso, y juego pintado en el suelo

-Para la parte principal de la sesión como en otras partes de la misma, nos aprovechamos de diferentes elementos que nos ofrece la zona de práctica de la clase. Entre ellos tenemos los juegos pintados en el suelo, los elementos arquitectónicos del centro como escaleras, columnas etc y las propias líneas de las pistas polideportivas; todo ello con el fin de no tener que usar más material, para automatizar algunas partes de la sesión como el calentamiento, y para introducir estos elementos dentro de los juegos o ejercicios que realizamos.

-También utilizamos estilos de enseñanza y estrategias en la práctica más tradicionales, relacionados con prácticas más analíticas que nos aseguran una actividad física más segura, por las características propias de estas intervenciones en las que la distancia entre alumnos es notoria, y en las que se ciñen a unas instrucciones cerradas y concretas.

-Usamos tizas de diferentes colores para la organización espacial de algunos juegos, lo que nos permite no tener que usar material extra, y con las que podemos crear espacios de una manera rápida y sencilla.

-Otra de las medidas de higiene será el lavado de manos tras el uso de un material; además de una u otra manera el alumnado se lavará las manos antes, durante y después de la sesión. También la toma de la temperatura a la entrada al centro escolar a las 9:00 horas; entrada que al igual que la salida se hace de manera gradual, en filas con distancia de seguridad e invirtiendo el tiempo necesario.

-La elección de organizaciones metodológicas que nos aseguran una distancia de seguridad y una individualización de la enseñanza.

\section{Resultados más relevantes}

Una vez recopilada toda la información anterior proponemos una unidad didáctica en la que trabajamos de manera global toda una serie de contenidos, que se adecúan de manera idónea al distanciamiento social y a las circunstancias sobre todo de temperatura que se dan en el mes de Julio. Con el objetivo esencial de asegurar las condiciones de seguridad y salud que se demandan,

\begin{tabular}{|c|c|c|}
\hline $\begin{array}{l}\text { Sesión 1: Juego sin material } 1 \\
\text { Tiempo: } 55^{\prime}\end{array}$ & \multicolumn{2}{|c|}{$\begin{array}{l}\text { Sesión 2: Juego sin material } 2 \\
\text { Tiempo: } 55^{\prime}\end{array}$} \\
\hline \multicolumn{3}{|c|}{ Objetivos de la sesión } \\
\hline -Desarrollar habilidades motrices básicas & \multicolumn{2}{|c|}{-Desarrollar habilidades motrices básicas } \\
\hline \multicolumn{3}{|c|}{ Información inicial } \\
\hline $\begin{array}{l}\text {-Información medidas COVID en espacio asignado en escaleras a cada } \\
\text { alumno/a con tiza. } \\
\text {-Aseo personal antes, durante y después de la sesión }\end{array}$ & \multicolumn{2}{|c|}{$\begin{array}{l}\text {-Información medidas COVID en espacio asignado en escaleras a cada } \\
\text { alumno/a con tiza. } \\
\text {-Aseo personal antes, durante y después de la sesión }\end{array}$} \\
\hline \multicolumn{3}{|c|}{ Calentamiento/Parte Inicial/Parte exploratoria } \\
\hline $\begin{array}{l}\text {-Analítico en círculo (movilidad articular, ejercicios de activación y } \\
\text { estiramientos) }\end{array}$ & \multicolumn{2}{|c|}{$\begin{array}{l}\text {-Calentamos en las huellas: desplazamientos por las huellas pintadas en } \\
\text { recorrido. }\end{array}$} \\
\hline \multicolumn{3}{|c|}{ Parte Principal } \\
\hline Medidas COVID & Descripción & Medidas COVID \\
\hline 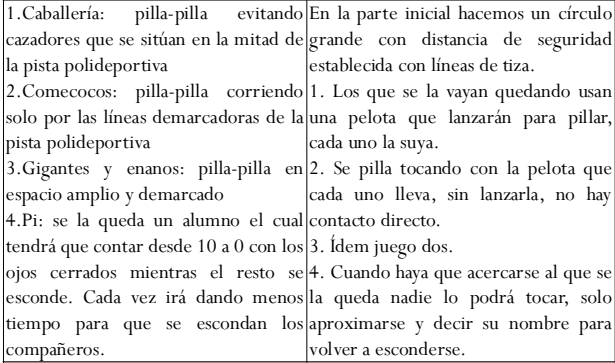 & $\begin{array}{l}\text { 1.Un, dos,tres pollito inglés: } \\
\text { juego de posición corporal. } \\
\text { 2.Los relevos: carreras } \\
\text { corriendo de frente, } \\
\text { corriendo de espaldas, a la } \\
\text { pata coja, saltos a pies } \\
\text { juntos... } \\
\text { 3.Cara cruz: en parejas } \\
\text { según diga el maestro cara o } \\
\text { cruz, se desplazarán } \\
\text { pillando unos a otros. }\end{array}$ & $\begin{array}{l}\text { 1. Distancia de seguridad entre compañeros. } \\
\text { 2. Se hacen } 3 \text { filas con distancia de seguridad } \\
\text { marcadas con tiza y con alineamiento entre } \\
\text { alumnado. El relevo lo marca el maestro, no } \\
\text { se pasan un testigo o se chocan las palmas. } \\
\text { 3. Distancia de seguridad entre cada pareja, } \\
\text { aumentamos la distancia para evitar choques } \\
\text { y disminuimos el espacio a correr unos } \\
\text { detrás de otros para limitar el juego a la } \\
\text { velocidad de reacción. }\end{array}$ \\
\hline \multicolumn{3}{|c|}{ Vuelta a la calma o Parte final } \\
\hline -El payaso: en circulo uno tratará de hacer reír al resto. & \multicolumn{2}{|c|}{ El espejo: juego estático de imitación } \\
\hline \multicolumn{3}{|c|}{ xión final } \\
\hline Los juegos de locomoción son un recurso motivador. & \multicolumn{2}{|c|}{$\begin{array}{l}\text { Las carreas de relevos deben asegurar un tiempo de recuperación entre } \\
\text { serie y serie. }\end{array}$} \\
\hline \multirow{2}{*}{\multicolumn{3}{|c|}{ Técnica de enseñanza, estrategia en la práctica, estilo de enseñanza }} \\
\hline & & \\
\hline Búsqueda, global, resolución de problemas. & Instrucción direct & ta, analítica pur \\
\hline \multicolumn{3}{|c|}{ Agrupamiento, espacio, participación y situación del docente } \\
\hline Gran grupo, pista polideportiva, masiva, externa. & \multicolumn{2}{|c|}{ Pequeños grupos, parejas, pista, alternativa, externa } \\
\hline \multicolumn{3}{|c|}{ Seguridad } \\
\hline $\begin{array}{l}\text { Lavado de manos entre cada uno de los juegos. Se asigna una pelota para cada } \\
\text { uno/a de uso exclusivo e individual. Desinfección del material en la mitad de } \\
\text { la sesión. Subidas y bajadas con mascarillas puestas. }\end{array}$ & \multicolumn{2}{|c|}{$\begin{array}{l}\text { En calentamiento y relevos distancia de seguridad en la fila, con } \\
\text { alineamiento y marcando cada posición de cada alumno/a con tiza. } \\
\text { Subidas y bajadas con mascarillas puestas. }\end{array}$} \\
\hline
\end{tabular}


Tabla 2 .

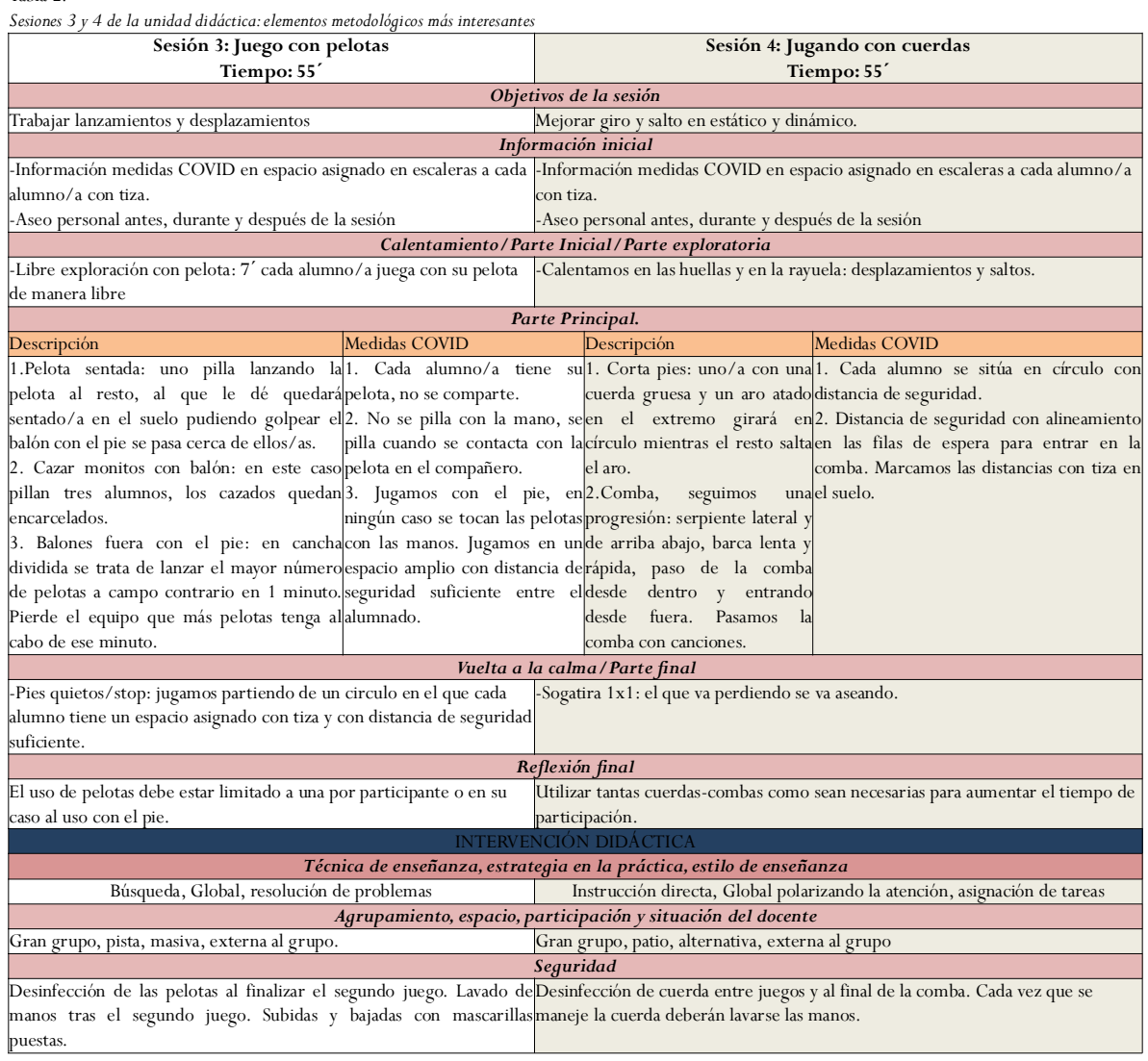

del maestro.

Algunas de las dificultades encontradas en el desarrollo de esta sesión fueron:

-La adaptación y modificación de la lógica interna de muchos de los juegos para adaptarlos a las características de salud y seguridad necesarias.

-Conseguir los automatismos en filas y escaleras.

- Evitar contactos y mantener una distancia de seguridad mínima en algunos juegos.

Algunas de las dificultades encontradas en el desarrollo de esta sesión fueron:

-Realizar las señalizaciones pertinentes y que estuvieran acordes a las ya nos atrevemos a lanzar una serie de sesiones que responden a esa necesidad, implementando medidas condeterminadas por el centro educativo.

-La pérdida de tiempo en la limpieza del material y cretas de seguridad en la gestión y uso de los recursos e instalaciones (Luis del Campo y SánchezPíriz, 2016), caracterizadas por estar sometidas a los cambios organizativos y metodológicos necesarios que aseguren la distancia social.

Las sesiones como último nivel de concreción curricular se basan en los trabajos de García y Ruiz (2001) y de Hernández y Sánchez (2002), de los cuales hemos extraído juegos, ideas y ejercicios, aparte de los propios aportados por la Junta de Andalucía en su material didáctico para primaria (2020) y las aportaciones
Tabla 3.

Sesiones 5 y 6 de la unidad didáctica: elementos metodológicos más interesantes

\begin{tabular}{|c|c|}
\hline $\begin{array}{l}\text { Sesión 5: Recorrido } \\
\text { Tiempo: } 55^{\prime}\end{array}$ & $\begin{array}{l}\text { Sesión 6: Jugando con aros } \\
\text { Tiempo: } 55^{\prime}\end{array}$ \\
\hline \multicolumn{2}{|c|}{ Objetivos de la sesión } \\
\hline \begin{tabular}{|l} 
Trabajar habilidades motrices básicas y perceptivas. \\
\end{tabular} & Trabajar habilidades motrices básicas con aros. \\
\hline \multicolumn{2}{|c|}{ Información inicial } \\
\hline $\begin{array}{l}\text {-Información medidas COVID en espacio asignado en escaleras a cada alumno/a con } \\
\text { tiza. } \\
\text {-Aseo personal antes, durante y después de la sesión }\end{array}$ & $\begin{array}{l}\text {-Información medidas COVID en espacio asignado en escaleras } \\
\text { a cada alumno/a con tiza. } \\
\text {-Aseo personal antes, durante y después de la sesión }\end{array}$ \\
\hline \multicolumn{2}{|c|}{ Calentamiento/Parte Inicial/Parte exploratoria } \\
\hline $\begin{array}{l}\text {-Elaboramos el recorrido con las propuestas del alumnado y con las premisas del } \\
\text { docente }\end{array}$ & $\begin{array}{l}\text {-Libre exploración con un aro: } 7^{\prime} \text { cada alumno/a juega con su } \\
\text { aro de manera libre }\end{array}$ \\
\hline \\
\hline $\begin{array}{l}\text { Parte Principal } \\
\text { Descripción }\end{array}$ & Aspectos organizativos \\
\hline \multicolumn{2}{|c|}{ 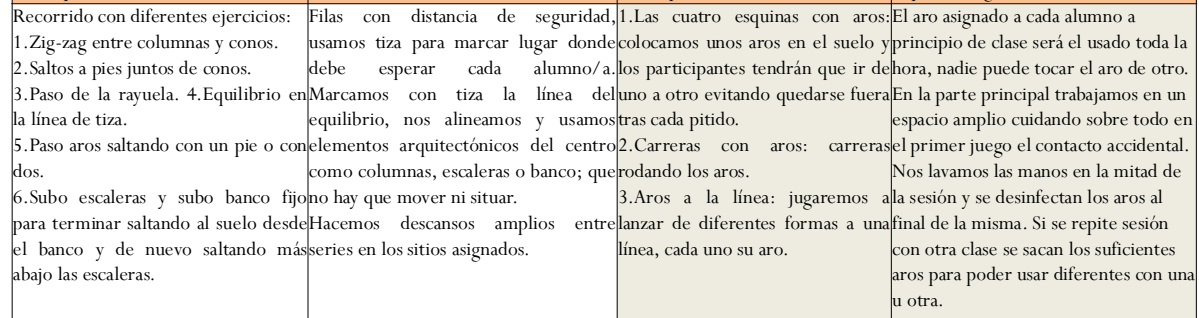 } \\
\hline \multicolumn{2}{|c|}{ Vuelta a la calma/Parte final } \\
\hline -Las películas: jugamos a hacer mímica de alguna película, serie o dibujos animados. & $\begin{array}{l}\text {-El baile del aro: el alumnado "baila" el aro con varias partes de } \\
\text { su cuerpo como el brazo, cintura... }\end{array}$ \\
\hline \multicolumn{2}{|c|}{ Reflexión final } \\
\hline Para aumentar el tiempo de compromiso motor se pueden plantear dos r & $\begin{array}{l}\text { Por las diferentes medidas a tomar el tiempo de compromiso } \\
\text { motor se reduce respecto a una sesión "ordinaria }\end{array}$ \\
\hline \multicolumn{2}{|c|}{ Técnica de enseñanza, estrategia en la práctica, estilo de enseñanza } \\
\hline Instrucción directa, analítica pura, asignación tareas & Instrucción directa, $\mathrm{g}$ \\
\hline \multicolumn{2}{|c|}{ Agrupamiento, espacio, participación y situación del docente } \\
\hline dividu & $\mathrm{Gr}$ \\
\hline \multirow[b]{2}{*}{ El material solo lo toca el maestro para colocarlo y para recoge } & \\
\hline & $\begin{array}{l}\text { Se utiliza el tiempo necesario para desinfectar los aros en mitad } \\
\text { de la sesión y para el lavado de manos }\end{array}$ \\
\hline
\end{tabular}


en el aseo del alumnado han afectado de manera directa al tiempo de compromiso motor.

-Dificultad para que nadie tocase un material que no es el asignado, y evitar contactos en los desplazamientos.

Algunas de las dificultades encontradas en el desarrollo de esta sesión fueron:

-Limpieza del material y lavado de manos en las sesiones.

-Preparación de las clases con el mínimo material posible.

-Mantener alta la motivación, dadas las características de las sesiones, con sus restricciones, las temperaturas elevadas y la propia dificultad por trabajar en época estival.

ción entre ellos.

Realizadas las sesiones enumeramos los resultados más destacados que podemos extraer de las mismas:

- Los juegos de desplazamiento son un recurso ideal aunque se necesitan espacios muy amplios de juego para evitar contactos involuntarios.

- Las carreas de relevos son un recurso ideal para trabajar con distanciamiento, aunque hay que remarcar al alumnado las normas en las filas.

- Saltar la comba resulta un trabajo adecuado aunque son necesarias tantas combas o cuerdas como grupos que aseguren la distancia social en un espacio amplio.

-El recorrido es un recurso muy útil, seguro y efectivo para mantener las distancias entre alumnado, aunque el compromiso moTabla 4.

\begin{tabular}{|c|c|}
\hline $\begin{array}{l}\text { Sesión 7: Circuito predeportivo: baloncesto } \\
\text { Tiempo: } 55^{\prime}\end{array}$ & $\begin{array}{l}\text { Sesión 8: Circuito predeportivo: balón-pie } \\
\text { Tiempo: } 55^{\prime}\end{array}$ \\
\hline \multicolumn{2}{|c|}{ Objetivos de la sesión } \\
\hline $\begin{array}{l}\text { Iniciar al alumnado en el trabajo de desarrollo de las habilidades motrices } \\
\text { genéricas y espećficicas }\end{array}$ & $\begin{array}{l}\text { Iniciar al alumnado en el trabajo de desarrollo de las habilidades motrices } \\
\text { genéricas y específicas }\end{array}$ \\
\hline \multicolumn{2}{|c|}{ Información inicial } \\
\hline -Ídem sesiones anteriores & -Ídem sesiones anteriores \\
\hline \multicolumn{2}{|c|}{ Calentamiento/Parte Inicial/Parte exploratoria } \\
\hline $\begin{array}{l}\text { Libre exploración: cada uno con su balón de baloncesto en cancha de } \\
\text { baloncesto }\end{array}$ & $\begin{array}{l}\text { Libre exploración: cada uno con su pelota de gomaespuma en pista } \\
\text { polideportiva }\end{array}$ \\
\hline \multicolumn{2}{|c|}{ Parte Principal } \\
\hline Medidas COVID & Medidas COVID \\
\hline \begin{tabular}{|l|l} 
Trabajamos en 7 estaciones, & En parte exploratoria incidimos er \\
minutos por estación; 2 series. & distancia de seguridad. Usamos tod \\
1.Sin salir del círculo central, & espacio. \\
manejo de balón con botes. & En el resto de ejercicios cada alur \\
2. En canasta tiro libre. & solo podrá usar su balón, asignad \\
3. Zig-zag botando. & principio de la clase. Se desinfectarán \\
4. En canasta tiro de mediab balones a mitad de sesión y se lavarán \\
distancia. & manos entre series. Aprovechamos lt \\
5. Lanzamientos de pecho, a una canastas. \\
mano, gancho... la pared. & Usamos tiza para acotar espacios. \\
6. En espacio manejo de balón sin & \\
bote. & \\
7. Entrada a canasta. &
\end{tabular} & 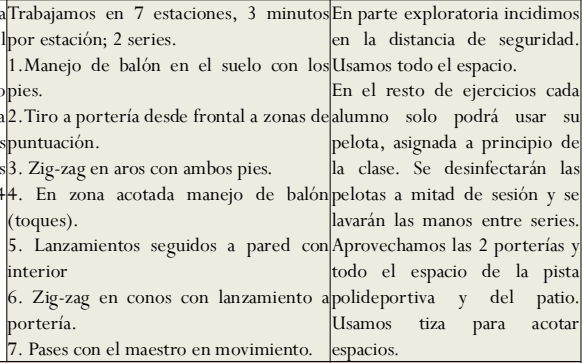 \\
\hline \multicolumn{2}{|c|}{ Vuelta a la calma/Parte final } \\
\hline \multicolumn{2}{|c|}{$\begin{array}{l}\begin{array}{l}\text { Concurso de tiro a canasta: desde varias posiciones y en las } 4 \text { canastas gana e el Futbolín: jugamos un partido en el que cada jugador tiene un espacio } \\
\text { que enceste desde todas las posiciones. } \\
\text { quado y del que no puede salir. }\end{array} \\
\text { Reflexión final }\end{array}$} \\
\hline \multicolumn{2}{|c|}{ Reflexión final } \\
\hline Para motivar el alumnado puede contar los logros que se van consiguiendo & $\begin{array}{l}\text { Para motivar el alumnado puede contar los logros que se van } \\
\text { consiguiendo. }\end{array}$ \\
\hline \multicolumn{2}{|r|}{ ica, estilo de enseñanza } \\
\hline Instrucción directa, analítica pura, asignación tareas & Instrucción directa, analítica pura, asignación tareas \\
\hline \multicolumn{2}{|c|}{ Agrupamiento, espacio, participación y situación del docente } \\
\hline Individual, pista polideportiva, simultánea, externa al grupo & Individual, pista polideportiva, simultánea, externa al grupo \\
\hline \multicolumn{2}{|c|}{ Seguridad } \\
\hline $\begin{array}{l}\text { Usamos todo el espacio que nos proporcionan las dos canchas de mini- } \\
\text { basquet y las } 4 \text { canastas. }\end{array}$ & $\begin{array}{l}\text { Usamos todo el espacio disponible. Se pueden acercar a beber agua de su } \\
\text { botella al sitio asignado en cualquier momento. Acotamos espacios con la } \\
\text { tiza y los conos. }\end{array}$ \\
\hline
\end{tabular}
tor se reduce. Podemos optar por realizar dos recorridos.

-Los aros es un material fácil de usar y que es muy útil para mantener el distanciamiento.

- El trabajo en circuito es ideal para mantener la distancia de seguridad, maximizar el tiempo de compromiso motor y motivar al alumnado.

Decisiones de acción para la próxima puesta en práctica

En base a las dificultades expuestas en cada sesión proponemos las siguientes soluciones:

-Tratamiento y planificación de cada actividad

-Elección de los contenidos a trabajar.

Algunas de las dificultades encontradas en el desarrollo de esta sesión fueron:

-La elaboración de unos objetivos que se relacionen de manera adecuada con los contenidos desarrollados, y que se adecúen a su vez a las necesidades de distancia y seguridad.

-Utilizar de manera dinámica estilos de enseñanza productivos con otros tradicionales en los que hay conteo, asignación de espacios predeterminados o una alinea- y ejercicio para minimizar riesgo de contacto

-Para automatismos en filas y escaleras: alineación con brazo y repetición hasta automatización

-Organización de los juegos por zonas o áreas para evitar contactos.

-Realizar las señalizaciones pertinentes en consonancia con el coordinador del programa de autoprotección del centro y con el coordinador «covid».

-Usar el mínimo material posible para evitar pérdidas de tiempo. 
-Tener controlado todo el material en una zona determinada, apartado.

-Orden predeterminado para lavarse las manos, evitando interrumpir la clase y sin tener que intervenir el profesor.

-Preparación de las clases con una búsqueda bibliográfica amplia.

-Mantener alta la motivación, alternando contenidos y variedad en los mismos; participación del alumnado en la elaboración de las sesiones.

-Selección de los contenidos en función de los contactos que puedan suceder y aplicar elementos metodológicos adecuados que ayuden.

-Seleccionar los criterios de evaluación y los estándares de aprendizaje que mejor se adecúen a las circunstancias.

-Priorizar estilos de enseñanza como la asignación de tareas, los individualizadores y la búsqueda guiada.

Finalmente y siguiendo los bloques de contenidos del currículum desarrollados en el Decreto 97/2015, y las propuestas de la Consejería de Educación de la Región de Murcia, proponemos una serie de contenidos o propuestas de trabajo que se pueden llevar a cabo respetando las medidas de seguridad propuestas:

Bloque 1. El cuerpo y sus habilidades perceptivomotrices

-Cuentos motores relacionados con centros de interés. En espacios amplios y evitando las concentraciones entorno al maestro.

-Juegos de orientación espacial. En espacios amplios.

-Trabajo en hileras en forma de relevos, respetando la distancia en las filas.

-Trabajo de velocidad gestual imitando gestos técnicos de cualquier deporte.

-Malabares. Las pelotas se adjudicarán a cada alumno/a, con desinfección entre sesiones y en espacios amplios para el feedforward y los feedbacks.

-Circuitos de habilidades. En estaciones suficientes, dobladas si es necesario y ocupando todo el espacio necesario.

-Recorridos de educación vial. En filas respetando la distancia de seguridad, si se utilizan implementos como bicis o patines, no se comparten, cada uno utiliza los suyos.

-Carreras de orientación: individuales en espacio muy amplio, con mucha información inicial, evitando coincidir en postas.

Bloque 2. La educación física como favorecedora de salud

-Estiramientos activos, movilidad articular y traba- jo de higiene postural. Individual con asignación a cada uno de un espacio concreto.

-Progresiones individuales del trabajo de equilibrio y control postural. En recorridos o circuitos, respetando la distancia de seguridad. Planes individuales.

-Trabajo de agilidad/flexibilidad individual. En recorridos o circuitos con distancia de seguridad y alineamiento en el caso de las filas.

-Ejercicios individuales de fuerza. Trabajo en circuito con espacios diferenciados, utilizando el mínimo material, con auto-cargas principalmente.

Bloque 3. La expresión corporal: expresión y creación artística

-Trabajo relacionado con la relajación sistemática como yoga o mindfulness.

-Juegos de mímica, juegos sensoriales auditivos y visuales, asegurando la distancia de seguridad, evitando los contactos.

-Dramatizaciones. En espacios amplios, en parejas o tríos.

-Coreografías de participación individual. Con participación alternativa y preparación más libre en espacios amplios.

-Juegos de percusión corporal individual

-Aerobic o body combat en formación geométrica.

Bloque 4. El juego y el deporte escolar

-Juegos de pistas y rastreo. En amplios espacios y con las pistas suficientes. Se pueden alternar los participantes. Evitar que toquen las pistas.

-Juegos de cancha dividida. Necesario tener varias pistas polideportivas para separar los jugadores. El material es personal y se desinfecta después de su uso.

-Juegos de blanco y diana. Utilización de material individual que se desinfectará tras su uso, espacios amplios y distancia de seguridad en las filas si es necesario.

-Juegos de campo y bate. Evitamos utilizar bate, usamos espacios amplios y dividimos los equipos para evitar exceso de fila.

-Juegos populares de participación alternativa. Principalmente participación alternativa, si es simultánea ampliar espacios y evitar encontronazos.

-Deportes individuales. En espacios asignados a cada uno, con material individual que se desinfectará tras uso. Se puede trabajar a través de circuitos.

-Deportes colectivos. Se trabajarán a través de circuitos de estaciones suficientes, con material individual, basándose en la técnica individual.

-Deportes alternativos. Dependiendo de sus características aplicaremos las medidas de los deportes individuales o de los colectivos. 


\section{Conclusiones}

En cuanto a las conclusiones, podemos determinar que se cumplió de manera adecuada con el objetivo inicial que se planteó, extrayendo de esta experiencia y con vistas a la próxima programación del curso 2020/ 21, una serie de medidas en la organización de las mismas que nos van a ayudar a cumplir con los protocolos de distancia y seguridad, dando consistencia a nuestra docencia y sobre todo priorizando la salud.

1. El primer y más destacado recurso metodológico que podemos usar durante el curso es la utilización de los circuitos para el trabajo eminentemente técnico en la iniciación a los deportes colectivos o para el desarrollo en progresión de cualquier contenido que adaptemos creativamente. También importante para individualizar la enseñanza, incluso pudiendo realizar planes individuales como estilo de enseñanza recurrente que se lleven a cabo en diferentes estaciones. Calderón y Palao (2005) señalan al respecto del uso organizativo en circuito que el tiempo de ejecución motora y el número de repeticiones realizadas de cada ejercicio es mayor en este tipo de organización respecto a otros como la organización en hileras los cuales requieren de más tiempo de espera. También los circuitos se perciben como más motivadores, favoreciendo el aprendizaje y el desarrollo de actitudes positivas hacia la práctica de actividad física, además y fundamentalmente haciendo la clase más eficaz aumentando el tiempo de práctica, como hemos comentado disminuyendo el tiempo de espera (Lozano y Viciana, 2003).

2. Otro recurso utilizado y que creemos que puede ser muy útil en el próximo curso escolar son los recorridos; principalmente para el trabajo de las habilidades motrices básicas y perceptivas, aunque con dos hándicaps, como pueden ser los tiempos de espera y la escasez de material que podemos usar. El objetivo de estos recorridos es motivar el movimiento de manera natural y espontánea entre los escolares (Martínez, Martí, Matas y Olivares, 2020) con aportaciones del alumnado, lo que le confiere un carácter creativo, en contraposición al espíritu analítico que también suelen acarrear; cuestión que por otro lado no censuramos en estas sesiones, ya que es necesario para mantener un orden, y sobre todo un distanciamiento social; aunque para nosotros lo que importa es si su aplicación mejora nuestra práctica habitual de enseñanza (Fajardo y García, s.f.). En lo que parece que no hay dudas como señala Martínez, Santaella y Rodríguez (2020) en el trabajo realizado con recorridos en ambientes de aprendizaje en EF, es en que el disfrute de los participantes fue alto en las sesiones que se organizaron mediante este recurso; suponemos que por la libertad de elección de actividades por parte de los participantes, y la percepción de autonomía.

3. En cuanto a los juegos en los que básicamente se trabajan los desplazamientos, ya sean pilla-pillas, relevos o cualquier otro tipo y que no requieren de material, podemos decir que aportan muchas variantes en cualquiera de las partes de la sesión; que la utilización ha sido positiva durante este programa estival y que hay que tener varios aspectos en cuenta para cerciorar la seguridad del alumnado. Por un lado el contacto directo queda prohibido, por lo que podemos usar algún tipo de material (pelotas, conos...) para que sea el que contacte (en pilla-pilla o en relevos con el testigo); por otro lado es fundamental que estos juegos se realicen en un espacio muy amplio en el que se eviten contactos, siendo importante también asegurar tiempos de descanso amplios. Como indica Valero (2008), estos juegos siempre estarán supeditados al cansancio del alumnado y al descenso motivacional en su desarrollo para terminarlos o pasar a otros.

Los relevos, al ser una prueba grupal de velocidad, cooperación y estrategia de equipo, supondrá una motivación para los alumnos por su aspecto competitivo, siempre desde un punto de vista educativo (Izquierdo, 2013); convirtiéndose en un recurso metodológico interesante, en el que al igual que en los recorridos deberán mantener la distancia de seguridad en las filas.

4. Dentro del material limitado por las circunstancias y de su uso funcional, optamos por las cuerdas, los aros y las pelotas de foam para el desarrollo de varias sesiones de esta unidad didáctica. La elección de este material es lógica por su accesibilidad y por la cantidad que generalmente suele haber en los centros educativos. Con este material hemos podido desarrollar diferentes tipos de juegos, actividades o ejercicios, siendo unos recursos que por sus características nos han facilitado el proceso de enseñanza-aprendizaje a la vez que nos han aportado variabilidad en la práctica, siempre teniendo en cuenta las medidas de desinfección y de uso necesarias en estos momentos. Tal y como señala Rivadeneyra (2001), la elección de este material debe estar en función de los criterios de polivalencia, adaptabilidad, manejabilidad, adaptabilidad, calidad o coste, respondiendo en nuestro caso a las necesidades actuales, por lo que creemos que serán unos recursos esenciales y básicos en nuestras clases de EF, aumentando la motivación y el entusiasmo del alumnado (Méndez-Giménez, de Ojeda-Pérez y Valverde-Pérez, 2016). 


\section{Referencias}

Alarcón-Meza, E. y Hall-López, J. (2020). Actividad física en estudiantes deportistas universitarios, previo y en el confinamiento por pandemia asociada al COVID-19 (Physical activity in university student athletes, prior and in confinement due to pandemic associated with COVID-19). Retos: Nuevas tendencias en Educación Física, Deporte y Recreación, (39), 572-575.

Aranceta-Bartrina, J., Gianzo-Citores, M., y Pérez-Rodrigo, C. (2020). Prevalence of overwight, obesity and abdominal obesity in the Spanish population aged 3 to 24 years. The ENPE study. Rev Esp Cardiol (Engl Ed), 73(4), 290-299. Doi:10.1016/jrec2019.07.023

Baena-Morales, S., López-Morales, J., y García-Taibo, O. (2020). La intervención docente en educación física durante el periodo de cuarentena por COVID-19 (Teaching intervention in physical education during quarantine for COVID-19). Retos, (39), 388 395.

Calderón, A. y Palao, J.M. (2005). Incidencia de la forma de organización en la sesión sobre el tiempo de práctica y la percepción de la motivación en el aprendizaje de habilidades atléticas. Apunts Educación Física y Deportes, 81,pp. 29-37. Institut Nacional d'Educació Física de Catalunya.

Cabrera, E.A. (2020). Actividad física y efectos psicológicos del confinamiento por covid-19. Revista INFAD de Psicología. International Journal of Developmental and Educational Psychology. , 2(1), 209-220.

Celis, C., Salas, C.,Yáñez, A. y Castillo, M. (2020). Inactividad física y sedentarismo. La otra cara de los efectos secundarios de la Pandemia de COVID-19. Revista médica de Chile, 148(6), 885886.

Consejería de Educación y Deporte (2020). Material didáctico de EF para primaria:programa de refuerzo estival. Junta de Andalucía.

Consejo General de la Educación Física y Deportiva (2020). Recomendaciones docentes para una educación física escolar segura y responsable ante la nueva normalidad. COLEF. Madrid.

Consejería de Educación de la Región de Murcia (2020). Medidas higiénico-sanitarias en las clases de educación física. Murcia, España.

Fajardo, M.T.y García, J. (s. f.). El empleo de ambientes de aprendizaje en Educación Física, para la enseñanza del equilibrio en primer curso de Educación Primaria.

Recuperado de https://www.um.es/c/document_library/ get_file?uuid $=05388198-408 \mathrm{c}-4$ a 8 c - b 8 d 0 faef8c3252e5\&groupId $=299436$.

Federación Andaluza de baloncesto (2020). Protocolo protección y prevención de la salud frente al COVID-19. Recuperado de http:/ /www.andaluzabaloncesto.org/. Sevilla, España.

García, E. y Ruiz, F. (2001). Propuestas de juegos con: globos, cuerdas, papeles, envases y saquitos. Madrid, España. Editorial Gymnos.

Hernández, A. y Sánchez, A. (2002). Aprendiendo a jugar. Sindicato Anpe. Murcia, España.

Hortigüela-Alcalá, D., Garijo, A., y Pérez-Pueyo, Ángel. (2021). La Educación Física en el contexto COVID-19. Un relato de profesores de diferentes etapas educativas (Physical Education in the COVID-19 context. A tale from teachers of different educational stages). Retos, 41, 764-774. https://doi.org/10.47197/ retos.v41i0.86368

Izquierdo, E. (2013). El modelo ludotécnico en la iniciación deportiva escolar. Programación de una unidad didáctica de relevos en atletismo (trabajo fin de grado). Universidad deValladolid, España.

Junta de Andalucía (2015a). Decreto 97/2015, de 3 de marzo. Recuperado de https://www.adideandalucia.es/normas/decretos/ Decreto97-2015EducacionPrimaria.pdf
Junta de Andalucía (2015b). Orden de 17 de marzo de 2015. Recuperado de http://www.juntadeandalucia.es/educacion/ descargasrecursos/curriculo-primaria/pdf/PDF/ textocompleto.pdf.

Lleixá,T., Capllonch, M. y González-Arévalo, C. (2015). Competencias básicas y programación de Educación Física. Validación de un cuestionario diagnóstico. Retos: nuevas tendencias en educación física, deporte y recreación, (27), 52-57.

Lozano, L. yViciana, J. (2003). Competencias docentes en educación física. Un estudio basado en la competencia de gestión del tiempo y la organización de la clase. En J.Viciana, Investigación en educación física y deportes. FCAFD. Universidad de Granada. España.

Luis del Campo,V.y Sánchez-Píriz, R. (2016). Análisis y evaluación de la seguridad de instalaciones y equipamientos deportivos escolares en la ciudad de Mérida (Extremadura). Retos: nuevastendencias en educación física, deporte y recreación, (29), 66-71.

Márquez, J. J. (2020). Inactividad física, ejercicio y pandemia COVID19. VIREF Revista de Educación Física, 9(2), 43-56.

Martínez, A. H., Martí, I. G., Matas,Y. y Olivares, S. C. (2020). Los Ambientes de aprendizaje en Educación física y Motivación en las primeras edades. Retos: nuevas tendencias en educación fisica, deporte y recreación, (38), 761-767.

McGuire D. K., Levine, B. D., Williamson, J.W., Snell, P. G., Blomqvist, C. G., Saltin, B. y Mitchell, J. H. (2001). A 30-year follow-up of the Dallas Bed Rest and Training Study:I. Effect of age on the cardiovascular response to exercise. Circulation, 104(12), 1350- 1357. doi:10.1161/ hc3701.096099

Méndez-Giménez, A., de Ojeda-Pérez, D. M. yValverde-Pérez, J. J. (2016).Valoración del alumnado y profesorado del material convencional y auto-construido: estudio longitudinal de diseño cruzado en Educación Deportiva. Retos. Nuevastendencias en Educación Física, Deporte y Recreación, (30), 20-25.

National Library of Medicine (2020). ClinicalTrials (SitioWeb). Recuperado de https: / / clinicaltrials.gov/

Pérez, F.J. (2020). Documento de apoyo al profesorado para evitar la propagación del coronavirus SARS-CoV-2 en un centro educativo. Recuperado de https: / /www.prevenciondocente.com

Rivadeneyra, M. L. (2001). Selección y optimización de recursos materiales favorecedores del desarrollo en la Educación Física. Lecturas: educación física y deportes, revista digital, 35.

Rivas, R.A. G., Gastélum-Cuadras, G., Velducea,W.V., Bustos, J. B. G., y Esparza, S. D. (2021). Análisis de la experiencia docente en clases de Educación Física durante el confinamiento por COVID19 en México (Analysis of teaching experience in Physical Education classes during COVID-19 confinement in Mexico). Retos, 42, 1-11.

Sánchez, J.A. O., del Pozo-Cruz, J., Rosa, R. M.A., Gómez, D. G., y Barbosa, F. Á. (2021). Efectos del sedentarismo en niños en edad escolar: revisión sistemática de estudios longitudinales. Retos: nuevas tendencias en educación física, deporte y recreación, (40), 7.

Sierra-Díaz, J., González-Víllora, S., Toledo-Guijarro, J.A., y Bermejo-Collada, C. (2021). Reflexiones sobre el proceso de enseñanza y aprendizaje en Educación Física durante la pandemia por COVID-19. Un caso real (Reflections on the teaching and learning process in Physical Education during the COVID-19 pandemic. A real case). Retos, 41, 866-878.

Zueck, M. D. C., García, A.A. R., Villalobos, J. M. R. y Gutiérrez, H. E. I. (2020). Satisfacción en las clases de Educación Física y la intencionalidad de ser activo en niños del nivel de primaria. Retos: nuevas tendencias en educación física, deporte y recreación, (37), 33-40. 